\title{
Progress in neuroscience in Africa: editorial
}

\author{
Fleur M. Howells $^{1}$ ・ V. A. Russell ${ }^{2}$
}

Published online: 11 January 2016

(C) Springer Science+Business Media New York 2016

\section{Content of editorial:}

This special issue highlights current research in basic and clinical neuroscience on the continent of Africa. Authors were invited to submit their work via established neuroscience networks in Africa including the Society of Neuroscientists of Africa (SONA). Our aim was to encourage junior through to senior neuroscience researchers to submit their work for consideration for publication in our special issue aimed at highlighting Progress in Neuroscience in Africa. We have, as in previous years, been delighted with the number of manuscripts submitted for peer review and congratulate the authors on their efforts.

The spectrum of papers in this special issue bears testimony to the development of neuroscience in Africa, increased research capacity, and expertise which may serve to promote international collaboration and further consolidate current international collaborations. Papers in this special issue address problems of sustainability of neuroscientific research in Africa, how this has been addressed in the past and how it should be approached in the future (Karikari et al. 2015; Quansah and Karikari 2015).

One of Africa's strengths is its wealth of medicinal plants. Traditional therapeutics studied in rodent models include Garcina kola and its neuroprotective qualities (Olajide et al. 2015), and the role of honey in preventing lead-induced behavioural changes and cognitive impairment by reducing oxidative stress (Abdulmajeed et al. 2015). Drugs of abuse, both local and

Fleur M. Howells

howellsfleur@gmail.com

1 Department of Psychiatry and Mental Health, University of Cape Town, Cape Town, South Africa

2 Department of Human Biology, University of Cape Town, Cape Town, South Africa

those obtained from outside of Africa which have had significant impact in Africa are featured. Khat, an indigenous psychostimulant, is shown to cause memory deficits in a rodent model (Kimani et al. 2015). Detrimental effects of methamphetamine are demonstrated in human studies showing frontal white matter changes and increased aggression in young adults (Lederer et al. 2015) and in an animal model of depression showing that early-life exposure to methamphetamine produces social and cognitive deficits in rats (Mouton et al. 2015). Human genetic research provides insight into adolescent abuse of alcohol and circadian clock genes (Dalvie et al. 2015b), and exposure to alcohol in utero is shown to decrease gray matter in human infants (Donald et al. 2015). In addition, neural and behavioural changes in rodents were assessed after treatment with nicotine-MDMA (Adeniyi et al. 2015).

Findings in rodent models of early life trauma include gender specific resistance to developing depression when exposed to maternal neglect (Dimatelis et al. 2015) and behavioural and biochemical changes following restraint stress in rats subjected to early life maternal separation (van Zyl et al. 2015). A potential pharmacological intervention, $\mathrm{d}$-cycloserine, is shown to facilitate fear extinction in rats (Malan-Muller et al. 2015). A meta-analysis of social and affective research in humans reveals key brain areas affected by testosterone (Heany et al. 2015).

Mental disorders that are both prevalent in the developing and developed worlds are investigated at the molecular level. These include the use of an animal model of maternal separation in a genetic rat model of attentiondeficit/hyperactivity disorder (ADHD), the findings reported show significant involvement of the major inhibitory and excitatory neurotransmitter systems (Sterley et al. 2015). Human genetic research in bipolar disorder shows the involvement of the hypothalamic-pituitary-adrenal axis (Dalvie et al. 2015a) and in obsessive compulsive 
disorder, polymorphisms within the neuronal cadherin gene are proposed to serve as a potential diagnostic marker (McGregor et al. 2015).

Our final group of papers addresses neurological disorders, these include a clinical case study that provides insight into the presentation of demyelinating disorders resembling multiple sclerosis in a patient previously treated for malaria (van Rensburg et al. 2015). Neuropsychological presentation of children with Sydenham's chorea and cognitive benefits of intravenous immunoglobulins are investigated (Gregorowski et al. 2015). Lastly, assessment of lifestyle risk factors was found to facilitate clinical interpretation of apolipoprotein $\mathrm{E}$ genotyping results in a subgroup of patients at risk for Alzheimer's disease (Luckhoff et al. 2015).
As can be seen from the composition of this special issue, innovation and development of novel therapeutics are driving current neuroscientific research in Africa. We look forward to future special issues that show the ever developing Progress in Neuroscience in Africa.

\section{Acknowledgments}

We thank the African Neuroscientists who made this special issue possible and look forward to their future submissions. Further we thank the many reviewers who supported and assisted authors by providing critical evaluation of their submitted works. 